\title{
Electron Beam-Plasma Interaction: Experimental and Computer Simulation Results
}

\author{
M. Virgínia Alves \\ Fábio do Prado \\ Renato S. Dallaqua

\begin{abstract}
Campos - SP - Brasil
\end{abstract} \\ Laboratório Associado de Plasma - Instituto Nacional de Pesquisas Espaciais, 12221-970 - São José dos
}

The excitation of Langmuir waves by a gentle bump-on-tail of the electron distributions has become the classic example of a kinetic instability. Clear manifestations of effects of this instability are observed in a number of space objects as solar wind, magnetotails, auroral regions and planetary foreshocks. The purpose of this work is to investigate the possible nonlinear phenomena associated with the Langmuir waves generated by the beam. This paper reports results obtained in a beam plasma experiment as well as some simulation results obtained using the particlein-cell code PDP1.

The interaction of an electron beam, with velocity $v_{b}$ and density $n_{b}$, with a higher density ambient plasma, $n_{0}$, produces unstable electrostatic waves with frequency near the plasma frequency, $\omega_{\mathrm{pe}}$, and wave number given by $\mathrm{k}_{0} \approx \omega_{\mathrm{pe}} / v_{\mathrm{b}}$. For a cold beam, in the limit $n_{b} / n_{0} \ll 1$, the instability is due to a feedback mechanism involving bunching of the electrons leading to a growth in the space charge density (hydrodynamic regime). A thermal spread $(\Delta v)$ in the velocities of the beam particles (kinetic regime) tends to suppress the hydrodynamic instability. This has an important implication even when the velocity spread is initially small because the development of the instability itself causes the velocity spread to increase. The condition for neglecting the beam thermal spread $(\Delta v)$ is $\left(n_{b} / n_{0}\right)^{1 / 3} \geq \Delta v / v_{b}[1,2]$.

The collective relaxation of the electron beam depends on the resonant interaction between the beam and the wave that it has excited. For small wave amplitude, $\mathrm{W}_{0} \ll\left(\mathrm{k}_{0} \lambda_{\mathrm{D}}\right)^{2}$, and warm beam case, quasilinear theory predictes that the beam relaxation occurs through "plateau" formation by flattening the beam distribution function $\left(f_{b}(v)\right)$ within a characteristic propagation distance, relaxation length $(l)$, that depends on $\left(\mathrm{n}_{\mathrm{b}} / \mathrm{n}_{0}\right)^{-1}$.

Saturation of the beam-plasma instability can also occurs via non-linear effects such as wave-wave interactions (e.g. [2], [3]). When the energy of the waves in resonance with the beam, $W_{r}$, becomes greater than a proper threshold, these waves become unstable to nonlinear instability. These instabilities act to transfer the field energy from wavelengths resonant with the beam to shorter wavelengths which are not resonants. When the energy transfer from $\mathrm{W}_{\mathrm{r}}$ exceeds the rate at which field energy is generated by the beam, the beam instability will be stabilized.
We use a double plasma device with multipole surface magnetic confinement [4]. Figure 1 shows a schematic diagram of the experimental apparatus. A discharge plasma is created by accelerating primary electrons produced by tungsten, hot cathode filaments in argon gas. The inner diameter (plasma diameter) of the device is $0.60 \mathrm{~m}$ and its total length is $1.20 \mathrm{~m}$. The chamber is divided into a source $\left(\mathrm{l}_{\mathrm{s}}=0.30 \mathrm{~m}\right)$ and a target chamber $\left(\mathrm{l}_{\mathrm{t}}=0.90 \mathrm{~m}\right)$ by two grids. The first one is connected to the multipole magnetic structure inside the source chamber and the other is connected to the structure of the target chamber. The beam is generated biasing the source grid negatively with respect to the grounded target grid. This electron beam propagates into the target chamber and ionizes the gas, thus creating a plasma in that chamber. Axially movable Langmuir probes are used to detect the wave signals and an electrostatic energy analyzer is used to measure the electron beam distribution function. Experiments are carried out in argon gas at filling pressure $(\mathrm{p})$ range $5 \times 10^{-5}<\mathrm{p}<5 \times$ $10^{-4} \mathrm{mbar}$. The typical parameters of the experiment are: $\mathrm{n}_{0} \sim 2 \times 10^{14}-8 \times{ }^{15} \mathrm{~m}^{-3}$, electron temperature $\mathrm{T}_{\mathrm{e}} \sim 1-3$ $\mathrm{eV}, \mathrm{T}_{\mathrm{e}} / \mathrm{T}_{\mathrm{i}} \gg 1, \Delta \mathrm{v} / \mathrm{v}_{\mathrm{b}}<0.3$, beam energy $\mathrm{E}_{\mathrm{b}} \leq 180 \mathrm{eV}$, and $5 \times 10^{-4}<\mathrm{n}_{\mathrm{b}} / \mathrm{n}_{0}<10^{-2}$.

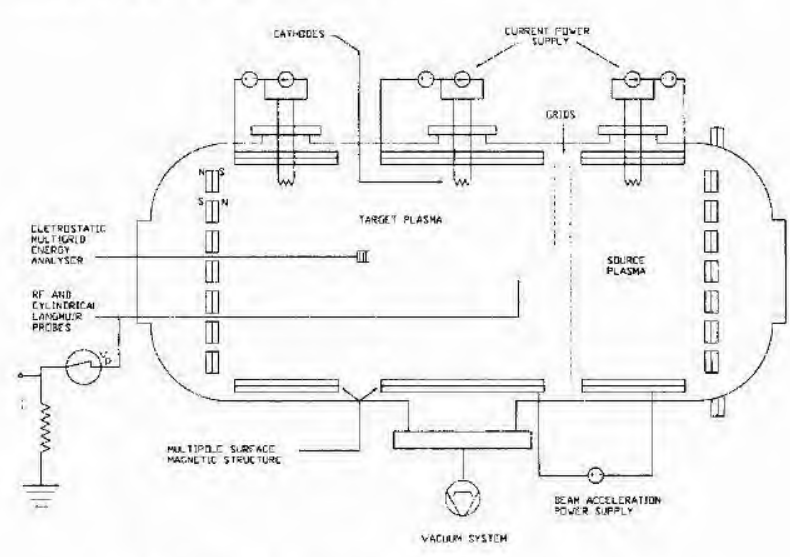

Fig. 1 - Schematic view of the PQUI device.

The ratio $n_{b} / n_{0}$ as well as the beam energy $E_{b}$ are important parameters in determining the beamplasma interaction evolution. We have explored three different beam energies, 60,120 e $180 \mathrm{eV}$, for $n_{b} / n_{0}$ in the range of $5 \times 10^{-4}$ to $10^{-2}$. 
First point we observe is that depending on the filling pressure, wich determines the ratio $n_{b} / n_{0}$, it is possible to have a cold or a warm beam. This is clearly observed in Figure 2 wich shows the ratio of measured frequency of the strongest peak of the unstable wave $\omega$ to plasma frequency $\omega_{p e}$, against $n_{b} / n_{0}$ for $E_{b}=180$ $\mathrm{eV}$. In the cold beam regime, as predicted by theory, $\omega$ $/ \omega_{\mathrm{pe}}<1[5]$. The observed limit value of $n_{b} / n_{0} \approx 7 \times 10^{-3}$ shown in the figure leads to a beam dispersion of approximately $\Delta v / v_{b} \approx 0.2$, wich is in agreement with our experimental conditions, $\Delta v / v_{b}<0.3$. One needs to notice that for $n_{b} / n_{0}<7 \times 10^{-3}$, the wave and energy growth are governed by kinetic effects [1].

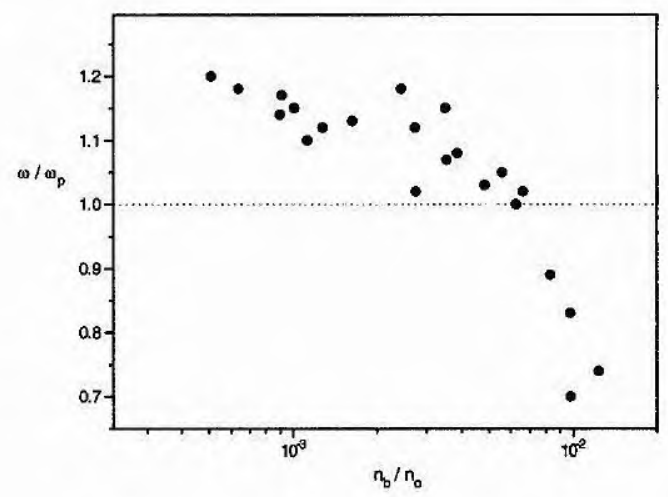

Fig. 2 - Plot of $\omega / \omega_{p}$ against $n_{b} / n_{0}$.

Fixing an energy beam we measure the position where the most unstable wave reaches its maximum amplitude, for several values of $n_{b} / n_{0}$. The results obtained for $\mathrm{E}_{\mathrm{b}}=180 \mathrm{eV}$ are shown in Figure 3 and they agree well with the ones predicted by quasilinear theory of beam relaxation, $\mathrm{l}_{\max } \propto\left(\mathrm{n}_{\mathrm{b}} / \mathrm{n}_{0}\right)^{-1}[6]$.

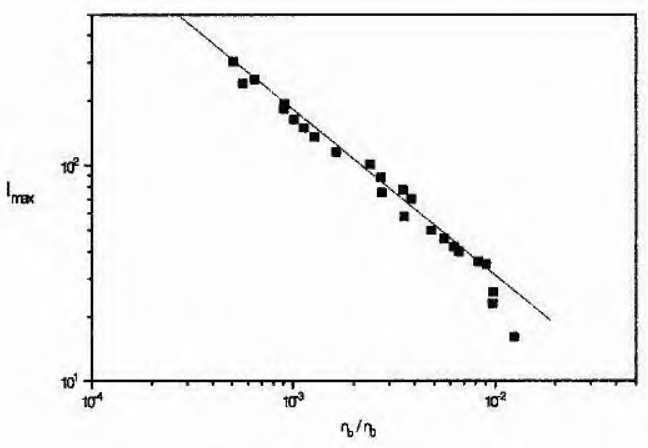

Fig. 3 - Plot of $\mathrm{l}_{\max }$ against $\mathrm{n}_{\mathrm{b}} / \mathrm{n}_{0}$

We have also obtained the space profile of the most unstable wave amplitude, at frequency $\omega \approx \omega_{\mathrm{p}}$ and also the space profile of second $\left(2 \omega_{\mathrm{p}}\right)$ and third $\left(3 \omega_{\mathrm{p}}\right)$ harmonic waves. Figures 4 (a)-(c) show the results. We observe an increasing of plasma wave amplitudes at distances $\mathrm{x}<1_{\max }$ and a decreasing of the amplitudes for $x>1_{\max }$, indicating that the beam plasma instability has been stabilized. We could expect to observe the presence of others growing waves or the acceleration of the bulk electrons. Analyses of the beam distribution function evolution will help to clarify this point. It is interesting that we do not observe a very localized region of high intensity waves, as has been recently observed experimentally [7]. Our experimental results also show that there is a density gradient in the target plasma. The shape and intensity of this gradient depend on the filling pressure and on the beam energy. Figure 5 shows the space profile of the target plasma density for $\mathrm{E}_{\mathrm{b}}=60,120$, and $180 \mathrm{eV}$ and $\mathrm{p}=2 \times 10^{-4}$ mbar.
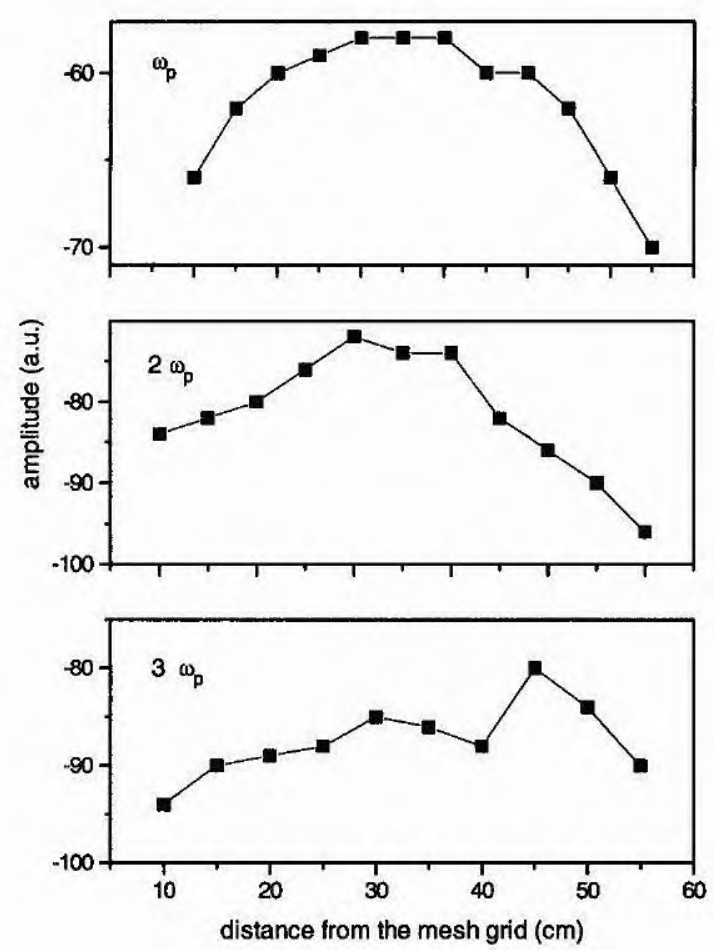

Fig. 4 - Space evolution of the wave amplitude at $\omega_{p}$, $2 \omega_{p}$ and $3 \omega_{p}$, observed in the experiment.

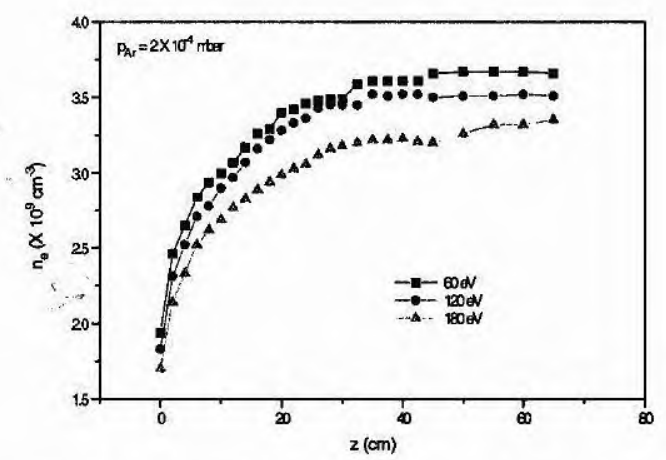

Fig. 5 - Space profile of the target plasma density for $\mathrm{E}_{\mathrm{b}}=60,120$, and $180 \mathrm{eV}$.

We investigated this point using the particlein-cell code PDP1 [8]. The simulation parameters are in the range of experimental ones. We simulate only the target chamber, the beam being continuously injected at the left boundary with a temperature of $2 \mathrm{eV}$. The potential is fixed at ground on both boundaries. Once the electrons reach the boundaries they are absorbed, 
contributing to the external surface charge. We introduced the following density gradient model: the initial density was increasing linearly from 0 at $\mathrm{x}=0$ to $2 \times 10^{14} \mathrm{~m}^{-3}$ at a position $\mathrm{x}_{0}$ and constant for $\mathrm{x}>\mathrm{x}_{0}$. We examined two cases, $x_{0}=0.1$ and $x_{0}=0.3 \mathrm{~m}$; the total length of the system was $0.8 \mathrm{~m}$. We observe that the position of the field spike is related with the spatial dimension of the gradient, but also, for a fixed $n_{b} / n_{0}$, it depends on $E_{b}$. The ratio $n_{b} / n_{0}$ for a fixed $E_{b}$, does not seem to play an important role. The simulations allow to investigate the several steps in the beam plasma interaction, while in the experiment, all measurements are done at times very large compared to the temporal growth rate of the beam plasma instability, or to the time for ion response which is very important to nonlinear phenomena. It is important to notice that for long runs, starting from a uniform density and including ionization, i.e., the plasma being continuously produced, a density gradient appears naturally, and it is not as strong as the one we have introduced, being more similar to the experimental results. It is possible to observe, besides the excitation of the Langmuir waves, the excitation of a low frequency wave. Figure 6 shows the space-time diagram of the ion density, normalized to $\mathrm{n}_{0}$, given by a gray scale with white as maximum. From this diagram it is possible to obtain the propagation velocity of the ion wave and it agrees well with the ion acoustic velocity. Also, the wave number obtained is of the oirder of $2 \mathrm{k}_{0}$, in agreement with the matching conditon of Langmuir pumping wave parametric decay in a backscattering Langmuir wave and an ion acoustic wave. As a result we observe the presence of standing waves together with lower-amplitude propagating waves. Figure 7 shows the space-time diagram of the high frequency electric field, given by a gray scale with the white as maximum. Figures 6 and 7 were obtained using as initial conditions: $\mathrm{E}_{\mathrm{b}}=180 \mathrm{eV}, \mathrm{n}_{\mathrm{b}} / \mathrm{n}_{0}=10^{-2}$, $\mathrm{n}_{0}=3 \times 10^{14} \mathrm{~m}^{-3}$, and $\mathrm{T}_{\mathrm{e}}=2 \mathrm{eV}$.

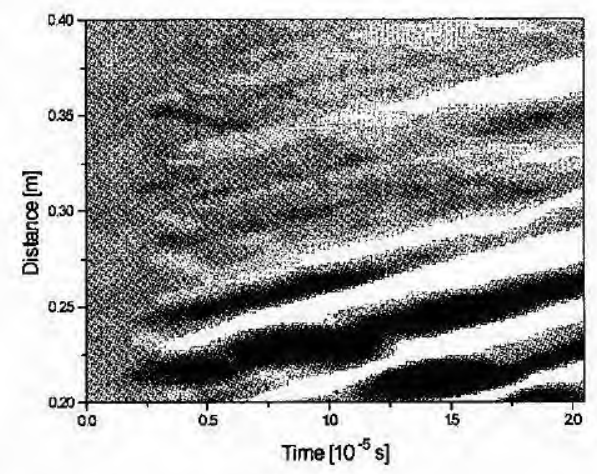

Fig.6 - Space-time diagram of ion density normalized to $\mathrm{n}_{0}$, given by a gray scale with white as maximum; $\mathrm{n}_{\mathrm{b}} / \mathrm{n}_{0}=10^{-2}, \mathrm{E}_{\mathrm{b}}=180 \mathrm{eV}$.

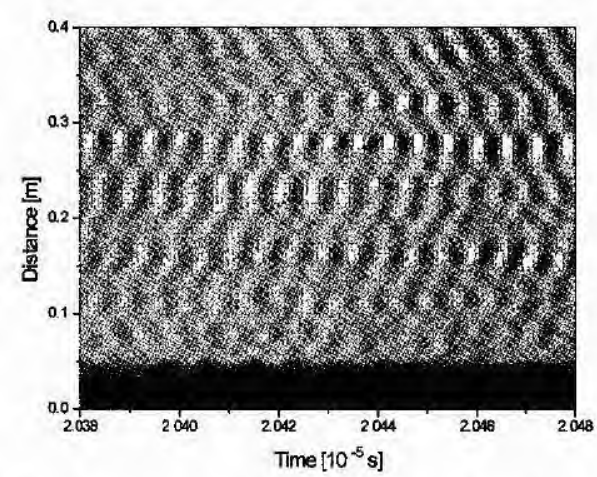

Fig. 7 - Space-time diagram of the electric field, given by a gray scale with white as maximum; $\mathrm{n}_{\mathrm{b}} / \mathrm{n}_{0}=10^{-2}, \mathrm{E}_{\mathrm{b}}=180 \mathrm{eV}$.

In conclusion, we obtained experimental results for the beam plasma interaction that present a good agreement with the theory. Investigation using particle-in-cell simulations shows the occurrence of wave-wave interaction, yet to be experimentally investigated.

This work was partially supported by Conselho Nacional de Desenvolvimento Científico e Tecnológico $(\mathrm{CNPq})$ and Fundação de Amparo à Pesquisa do Estado de São Paulo (FAPESP).

\section{REFERENCES}

D. B. Melrose Instabilities in Space and Laboratory Plasmas (Cambridge University Press, 1986), Chap. 3,4 .

H.L. Rowland, Phys. Fluids, 23, 508 (1980).

K. Papadopoulos, Comments Plasma Phys. Controlled Fusion, 9,11, (1984).

J. L. Ferreira, F. do Prado, M. V. Alves, E. D. Campos and D. M. Karfidov, Revista de Física Aplicada e Instrumentação, 9, 47 (1994).

N. Hayashi, M. Tanaka, S. Shinohara, and Y. Kawai, Phys. Plasmas, 2, 3582 (1995).

A. A. Gallev, R. Z. Sagdeev, V. D. Shapiro and V. I. Shevchenko, Sov. Phys. JETP, 45, 266 (1977).

H. Gunell, J. P. Verboncoeur, N. Brenning, and S. Torven, Phys. Review Letters 77, 5059 (1996).

J. P. Verboncoeur, M. V. Alves, V. Vahedi, and C. K. Birdsall, J. Comput. Phys.104, 321 (1993). 